Check for updates

Cite this: Chem. Commun., 2022, 58,3629

Received 9th December 2021, Accepted 15th January 2022

DOI: $10.1039 / \mathrm{d} 1 \mathrm{cc} 06936 \mathrm{e}$

rsc.li/chemcomm

\section{Mismatch binding ligand upregulated back- splicing reaction producing circular RNA in a cellular model $\dagger$}

\author{
Lu Ni, (D) Takeshi Yamada, (D) Asako Murata (D) and Kazuhiko Nakatani (D) *
}

\begin{abstract}
Circular RNA (circRNA) is a covalently closed single-stranded RNA produced from pre-mRNAs via back-splicing reaction, an alternative form of splicing. Here, we show naphthyridine carbamate dimer (NCD) upregulating the production of a circRNA from a pre-mRNA containing NCD-binding site UGGAA/UGGAA in cells, demonstrating the feasibility of small-molecule mediated circRNA production.
\end{abstract}

CircRNAs are covalently closed single-stranded RNA, without $5^{\prime}$ cap or $3^{\prime}$ polyA tail. ${ }^{1,2}$ Although circRNAs were initially considered by-products of aberrant splicing, ${ }^{3,4}$ they have recently received a surge of interest due to their ubiquitous presence in various cell types and tissues across many eukaryotic organisms. ${ }^{5-10}$ CircRNAs can act as miRNA sponges, ${ }^{10-12}$ competitive endogenous RNAs for RNA-binding proteins, ${ }^{13,14}$ competitors to canonical splicing, ${ }^{13}$ and translation templates. ${ }^{15}$ Consequently, circRNA dysregulation has become a major focus for the study of disease pathology, as they are implicated in various diseases, especially cancers ${ }^{11,16,17}$ and neurological disorders. ${ }^{10,18,19}$

CircRNAs are produced by a unique biogenesis mechanism: back-splicing. Mature mRNAs are produced from pre-mRNA by linear splicing initiated by the attack of the $2^{\prime}$-hydroxyl group of the branch-point adenosine (BPA) to the phosphodiester of $5^{\prime}$ splice site ( $\left.5^{\prime} \mathrm{ss}\right)$ in the same intron (Fig. 1A, dotted line). ${ }^{20}$ The majority of circRNAs, in contrast, is produced by back-splicing, where the initial attack of BPA's $2^{\prime}-\mathrm{OH}$ occurs at the $5^{\prime}$ ss of the downstream intron (Fig. 1A, solid line, step a). The produced $3^{\prime}$ $\mathrm{OH}$ group of the exon then attacks the $3^{\prime}$ ss in the upstream intron (step b), forming a covalently closed circRNA. ${ }^{21}$ Most notably, this back-splicing process is facilitated by base pairing between reverse complementary matches (RCMs, Fig. 1A, yellow arrows) in introns flanking the circularizing exon. ${ }^{22,23}$ RCMs are highly complementary sequences acting as a

SANKEN (The Institute of Scientific and Industrial Research), Osaka University, 8-1 Mihogaoka, Ibaraki 567-0047, Japan. E-mail: nakatani@sanken.osaka-u.ac.jp $\dagger$ Electronic supplementary information (ESI) available. See DOI: 10.1039/ d1cc06936e

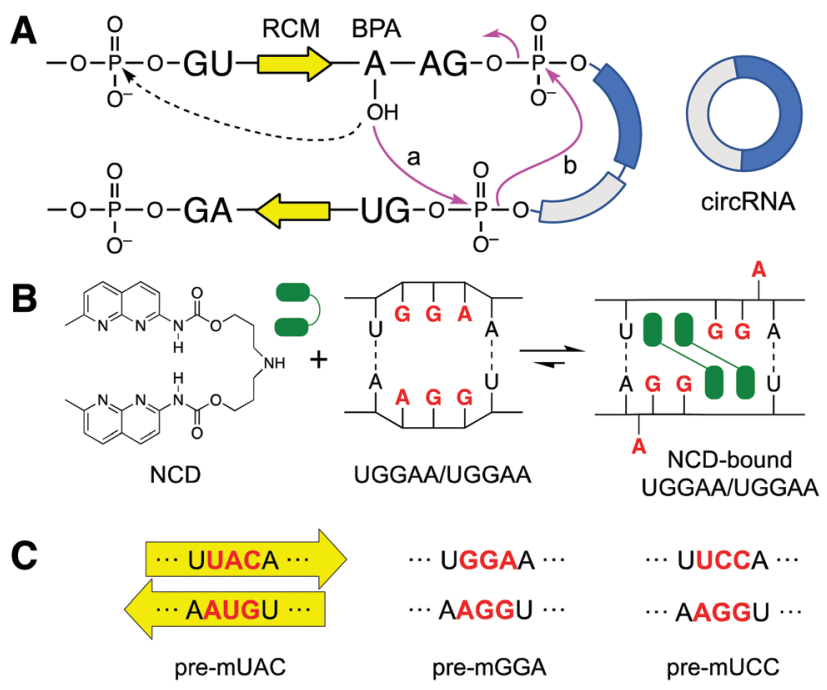

Fig. 1 (A) Schematic representation of back-splicing (solid pink arrows) to form circRNA and the initial step for the linear splicing (dotted arrow). (B) Chemical structure of NCD and illustration of NCD binding to $5^{\prime}$-UGGAA$3^{\prime} / 5^{\prime}$-UGGAA-3'. (C) The original RCMs' sequence UAC/GUA (pre-mUAC) was mutated into GGA/GGA (pre-mGGA) and UCC/GGA (pre-mUCC), the former of which contains the NCD binding site.

cis-regulatory element, forming base pairs between themselves to bring the BPA and the $5^{\prime}$ ss of the downstream intron close together. ${ }^{16,24}$ Bioinformatic analysis further revealed that distinct complementary repeat sequences play the role of RCMs as they are commonly found flanking circularizing exons. ${ }^{25-27}$

We have developed small molecules binding to mismatched base pairs in RNAs as mismatch binding ligands (MBLs) to regulate the functions of RNAs by facilitating the formation of ligand-stabilized structures. ${ }^{28,29}$ In this study, we hypothesized that MBLs could upregulate circRNA production by bringing the BPA's $2^{\prime}-\mathrm{OH}$ in close vicinity of the downstream $5^{\prime}$ ss.

We recently reported that one of the MBLs, NCD (Fig. 1B), forms a 2:1 complex with $5^{\prime}$-UGGAA- $3^{\prime} / 5^{\prime}$-UGGAA- $3^{\prime}$ motif (hereafter as UGGAA/UGGAA) in duplex RNAs. ${ }^{30}$ The complex 
structure was determined by NMR, which revealed the binding of two NCD molecules to four guanines of the internal loop present in the UGGAA/UGGAA with a concomitant flipping out of the adenine bases (Fig. 1B). Here, we report that NCD binding to the engineered RCMs involving a UGGAA/UGGAA motif increased the production of circRNA from exons flanked by those RCMs in HeLa cells. The effect of NCD is sensitively dependent on the presence of the UGGAA/UGGAA motif and NCD concentration, showing the increased formation of circRNA is due to the proximal location of BPA to the $5^{\prime}$ ss in backsplicing. We show that circRNA could be regulated by small molecules, offering new opportunities to study their biological functions.

Before starting cellular model experiments, a surface plasmon resonance (SPR) assay was conducted to confirm NCD binding to GGA/GGA. 5'-Biotinylated hairpin RNA containing either a GGA/ GGA mismatch or a control full match (UCC/GGA) was separately immobilized on a streptavidin-coated sensor chip. (Fig. 2A) The SPR responses were then measured at different concentrations of NCD. In addition to NCD, QCD, an NCD analog lacking binding ability towards GGA/GGA, was also studied. As shown in Fig. 2B, NCD showed a dose-dependent response to GGA/GGA-containing RNA (red line), which yielded an apparent equilibrium dissociation constant $\left(K_{\text {dapp }}\right)$ of $1.29 \mu \mathrm{M}$. In contrast, NCD showed a weak response to UCC/GGA-containing RNA (blue line) (Fig. 2B left). On the other hand, QCD showed negligible responses to both hairpin RNA (Fig. 2B right). These results showed the selective and robust binding of NCD to RNA containing the GGA/GGA mismatch site and the lack of binding of QCD to either mismatch or fully matched sequence.

The plasmids used for cellular experiments were designed according to Scheme 1. We used a circRNA expression construct

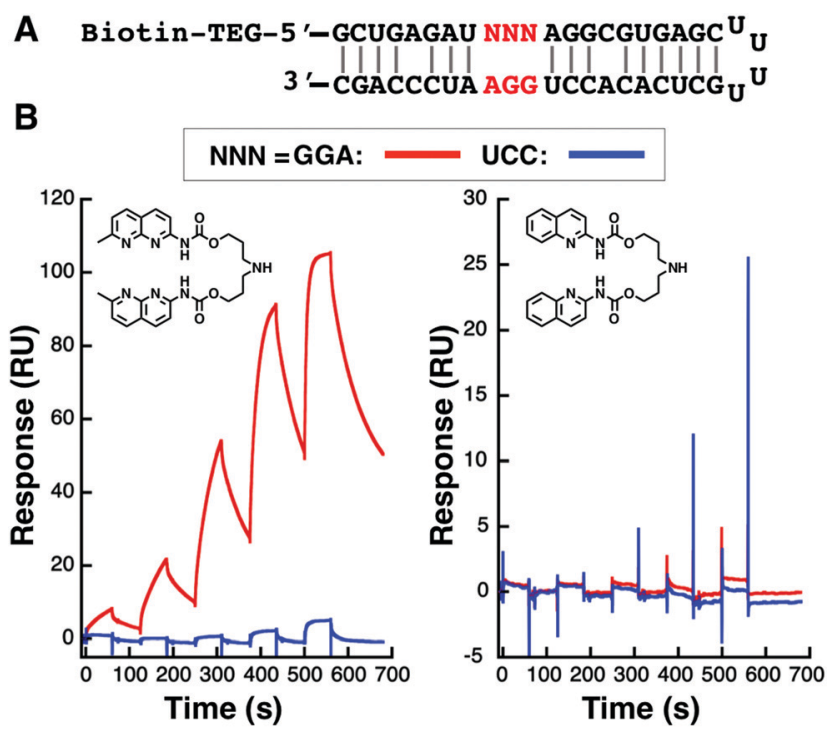

Fig. 2 SPR experiments to determine the affinity and selectivity of NCD and QCD towards immobilized target and control sequences. (A) The sequence of the immobilized hairpin RNA containing GGA/GGA and UCC/ GGA. (B) SPR response of GGA/GGA (red) and full match UCC/GGA (blue) RNA in the presence of NCD (left) and QCD (right).

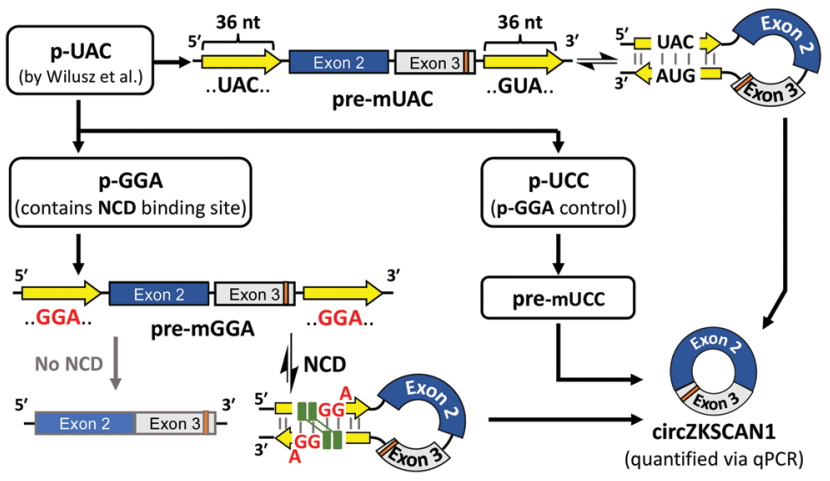

Scheme 1 Schematic representation of plasmid designs and general flow of the experiment.

(hereon denoted as p-UAC) coding for a pre-mRNA (pre-mUAC in Fig. 1C. The whole sequence is shown in Fig. S1B, ESI $\dagger$ ) previously reported by Wilusz et al. to produce a well-studied circRNA, circZKSCAN1 (stemming from exon 2 and 3 of host gene: ZKSCAN1). ${ }^{17,22}$ Pre-mUAC contains a pair of 36-nt RCMs (shown as yellow arrows in Fig. 1A and C), which was determined to be the minimum length necessary for producing circZKSCAN1 via back-splicing reaction. We then introduced the NCD-binding site in the RCMs of pre-mUAC by changing an original UAC/GUA site in the RCM of pre-mUAC to GGA/GGA, resulting in a construct coding for NCD-responsive pre-mRNA, denoted as pre-mGGA. (Fig. 1C) In addition, pre-mUCC, where a full-match sequence UCC/GGA was introduced into the same position, was designed as a control sequence of pre-mGGA (Fig. 1C and Fig. S1A, ESI $\dagger$ ). Hereon, plasmids coding premGGA and pre-mUCC would be denoted as p-GGA and p-UCC, respectively (sequences in Fig. S1C and D and table showing the corresponding transcribed RNA is shown in Fig. S1A, ESI $\dagger$ ). The introduced mismatch in pre-mGGA is expected to reduce the thermal stability of the base-paired complementary sequences, resulting in reduced back-splicing efficiency. ${ }^{22}$ However, binding of NCD to the mismatch site would stabilize the hairpin structure and thus can recover the efficiency loss in backsplicing and circZKSCAN1 production.

To investigate the effect of NCD on the upregulation of circZKSCAN1 production from cells expressing either premGGA or pre-mUCC in the cellular environment. HeLa cells transfected with either p-GGA or p-UCC were treated in the presence of various concentrations of NCD or QCD. The expression levels of circZKSCAN1 were then determined by reverse transcription-quantitative PCR (RT-qPCR) $)^{1,31}$ using a "divergent" primer which amplifies across the back-splice junction (shown as a triangle in Fig. 3A) to detect the circZKSCAN1.

We first confirmed the selectivity of our primer design in detecting circZKSCAN1 stemming from either p-GGA or p-UCC in the presence of endogenous circZKSCAN1. We took advantage of a single nucleotide difference present in the ZKSCAN1 sequence of p-GGA and p-UCC (Shown in orange in Fig. 3A), where thymine in exon 3 of endogenous ZKSCAN1 gene was cytosine (The $\mathrm{C}$ is highlighted in red in Fig. S1, ESI $\dagger$ ). The $3^{\prime}$ terminus of the forward primer (circZKSCAN1_Fw in Table S1, 
A

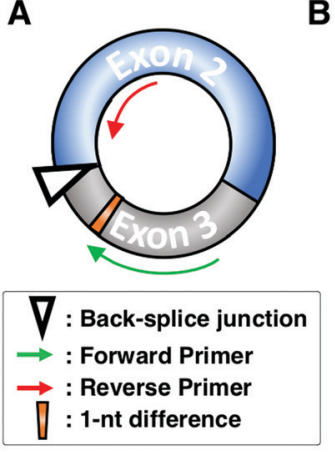

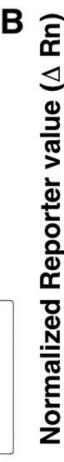

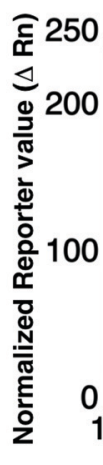

Fig. 3 (A) Schematic representation of circZKSCAN1. The site of back splice-junction is shown with a triangle, the green and red arrows represent the primers used for the $\mathrm{QPCR}$, and the orange highlight represents the location of $1 \mathrm{nt}$ difference between circZKSCAN1 and its endogenous counterpart, and (B) the RT-qPCR amplification curves of beta-actin and circZKSCAN1 using the designed primers. Each amplification curve is represented as follows: beta-actin in non-transfected HeLa (dotted line), beta-actin in p-GGA transfected cell (black line), circZKSCAN1 in nontransfected HeLa (green line), circZKSCAN1 in p-GGA transfected HeLa (red line), circZKSCAN1 in p-GGA transfected HeLa in the presence of $5 \mu \mathrm{M}$ NCD (blue line).

ESI $\dagger$ ) was set to end at the 1-nt difference to selectively amplify circZKSCAN1 stemming from these plasmids (Fig. 3A and Fig. S1, ESI $\dagger) .{ }^{32}$ The specificity of the divergent primer towards circZKSCAN1 stemming from these plasmids was confirmed by comparing the RT-qPCR amplification curves of the nontransfected HeLa cells against HeLa cells transfected with p-GGA treated with 0 or $5 \mu \mathrm{M}$ NCD (Fig. 3B and Table 1). While the difference in cycle threshold $\left(C_{\mathrm{t}}\right)$ values for the internal standard, beta-actin: a housekeeping gene, showed similar values for both non-transfected (black line) and p-GGA transfected cells (dotted line) $(19.5,20.0$, respectively), confirming that the same amount of total RNAs were used for analysis. The non-transfected HeLa cells (green line) showed a 7.2 cycle difference in circZKSCAN1 $C_{\mathrm{t}}$ value compared to those transfected with p-GGA (red line). In summary, PCR amplification of circZKSCAN1 stemming from p-GGA is approximately 147 -fold more efficient $\left(2^{-7.2}=1 / 147\right)$ than endogenous circZKSCAN1. Hereon all descriptions regarding circZKSCAN1 refer to circZKSCAN1 stemming from p-GGA or p-UCC.

The specificity of the divergent primer towards circZKSCAN1 was further confirmed by polyacrylamide gel electrophoresis (PAGE) of the amplified products obtained from p-GGA transfected cells treated with 0 and $5 \mu \mathrm{M}$ NCD, and $5 \mu \mathrm{M}$ QCD

Table 1 Comparison between the calculated $C_{t}$ values of HeLa only and p-GGA transfected cells (0 and $5 \mu$ M NCD). (Std. Dev. = standard deviation, $n=3$ )

\begin{tabular}{lcc}
\hline Samples & \multicolumn{2}{c}{ Average $C_{\mathrm{t}}$ Std. Dev. } \\
\hline p-GGA transfected HeLa beta-actin & 19.5 & 0.16 \\
HeLa only beta-actin & 20.0 & 0.14 \\
p-GGA transfected HeLa circZKSCAN1 (5 $\mu$ M NCD) & 24.6 & 0.38 \\
p-GGA transfected HeLa circZKSCAN1 (0 $\mu$ M NCD) & 27.2 & 0.30 \\
Non-transfected HeLa circZKSCAN1 & 34.4 & 1.1
\end{tabular}

(Fig. S2A, ESI $\dagger$ ). Only a single DNA band of the expected size (approx. $140 \mathrm{bp}$ ) was detected on the gel under each condition. Moreover, the sequencing of the PCR products, obtained from the cells transfected with p-GGA or p-UCC, showed the backsplicing junction sequence (Fig. S2B, ESI $\dagger$ ), corroborating the suitability of our divergent primer for detecting circZKSCAN1.

Additionally, to confirm the circular form of the detected circZKSCAN1, total RNA was treated by Ribonuclease R (RNase R) prior to the RT-qPCR experiment (Fig. S3, ESI $\dagger$ ). ${ }^{25}$ The $R_{\mathrm{Q}}$ of circZKSCAN1 showed a significantly higher resistance to RNase $\mathrm{R}$ digestion than beta-actin: a linear mRNA, further confirming the circular form of the detected circZKSCAN1.

The comparative $C_{\mathrm{t}}\left(\Delta \Delta C_{\mathrm{t}}\right)$ method $^{33}$ was used to determine the relative quantity $\left(R_{\mathrm{Q}}\right)$ of circZKSCAN1 in the presence of different concentrations of ligands. The raw $C_{\mathrm{t}}$ of circZKSCAN1 were first normalized against mRNA of beta-actin to give a $\Delta C_{\mathrm{t}}$ value. The $\Delta C_{\mathrm{t}}$ values of treated samples were then further normalized against those in the absence of ligands $(\mathbf{N C D}=0 \mu \mathrm{M})$ to provide $\Delta \Delta C_{\mathrm{t}}$. Then the $R_{\mathrm{Q}}$ value was calculated from the obtained $\Delta \Delta C_{\mathrm{t}}$ value $\left(R_{\mathrm{Q}}=2^{-\Delta \Delta C_{\mathrm{t}}}\right)$. (All $C_{\mathrm{t}}$ and $\Delta \Delta C_{\mathrm{t}}$ values used for calculations of $R_{\mathrm{Q}}$ are shown in $\left.\mathrm{ESI} \dagger\right)$.

Cells transfected with p-GGA, expressing pre-mGGA, showed a concentration-dependent increase in the $R_{\mathrm{Q}}$ of circZKSCAN1 in the presence of NCD. Where cells treated with $3 \mu \mathrm{M}$ NCD showed an apparent 3.1-fold increase in $R_{\mathrm{Q}}$ of circZKSCAN1 ( $p$ $<1 \times 10^{-6}$ ), while at the highest concentration of $5 \mu \mathrm{M} \mathrm{NCD,} \mathrm{a}$ 5.6-fold increase was observed $\left(p<1 \times 10^{-6}\right.$, Fig. 4A). In contrast, no statistically significant change in $R_{\mathrm{Q}}$ was observed for cells treated with $5 \mu \mathrm{M}$ QCD. On the other hand, the cells transfected with p-UCC, expressing pre-mUCC, the fully matched counterpart to pre-mGGA, were also treated similarly with the ligands (Fig. 4B). However, no statistically significant change in $R_{\mathrm{Q}}$ of circZKSCAN1 was observed either upon NCDor QCD- treatment of the cells expressing p-UCC.

Additionally, the changes in endogenous circRNAs in NCD presence were also studied. Here, the expression changes of four circRNAs (circHIKP3, circEPHB4, circFOXO3, and circPVT1) were investigated in the presence of 3 or $5 \mu \mathrm{M}$ NCD
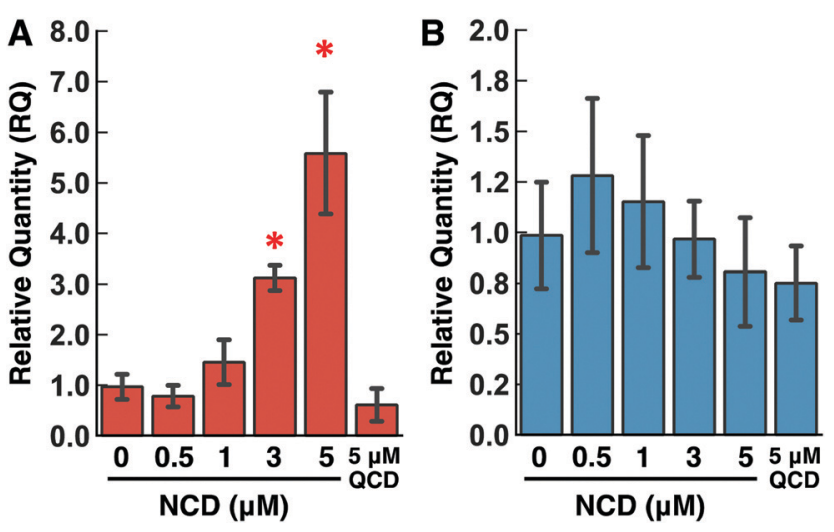

Fig. 4 NCD concentration-dependent circZKSCAN1 change for HeLa cells transfected with either (A) p-GGA or (B) p-UCC ${ }^{*}: p<0.05$, fold change $\geq 2$; the significance was obtained against the sample treated with $0 \mu \mathrm{M}$ NCD, using two-tailed $t$-test, $n=7$ ). 
concentration and $5 \mu \mathrm{M}$ QCD. As shown in Fig. S4 (ESI $\dagger$ ), we did not observe the induced circRNA formation of the above four endogenous circRNAs for both p-GGA and p-UCC transfected cells. However, we observed down-regulation of circEPHB4 under $5 \mu \mathrm{M}$ NCD in both cases. While we searched for potential NCD binding sites within 1000 bp up/downstream intron of the circEPHB4 transcript within its host pre-mRNA, we could not identify any relevant features which may cause the fluctuations mentioned above in expression level.

Finally, to determine the extent of NCD's capacity to induce recovery in circZKSCAN1 expression for pre-mGGA, $R_{\mathrm{Q}}$ of circZKCAN1 for cells transfected with p-GGA were calculated relative to circZKCAN1 amount in the cells transfected with the control p-UCC (NCD $0 \mu \mathrm{M}$, Fig. S5, ESI $\dagger$ ). The results show that in the absence of NCD, cells transfected with p-GGA showed $R_{\mathrm{Q}}$ of $0.074\left(p<3 \times 10^{-6}\right)$ relative to $\mathbf{p}$-UCC transfected cells, indicating a significant reduction in the production of circZKSCAN1 with the introduction of GGA/GGA mismatches into the RCMs. However, in the presence of $5 \mu \mathrm{M} \mathrm{NCD}$, the cells transfected with p-GGA showed an $R_{\mathrm{Q}}$ of 0.41 (p $<0.005$ ), suggesting that under the highest concentration of $5 \mu \mathrm{M}, \mathrm{NCD}$ was capable of inducing $34 \%(0.41-0.074=0.34)$ recovery in the expression of circZKSCAN1 for pre-mGGA, with regards to pre-mUCC.

In conclusion, the results of the in-cell experiments shown above are consistent with the SPR results shown in Fig. 2B. The apparent increase in circZKSCAN1 production likely follows the hypothesized mechanism, where the NCD binding to the UGGAA/UGGAA internal loop in RCMs facilitated the increase in circZKSCAN1 production by stabilizing RCMs and bringing the BPA to the proximity of $5^{\prime}$ ss. In this paper, we have demonstrated that an RNA-binding small molecule can upregulate circZKSCAN1 production in the cellular model by using our rationally designed molecule NCD and engineered construct containing an NCD-binding motif in its introns. Moreover, RCMs and repeat sequences found in introns adjacent to exons forming circRNAs have many unpaired regions, such as stem-loops and bulge loops, when they form duplex structures. These unpaired regions in dsRNA provide potential small molecule binding sites. Thus, the demonstrated model provides a stepping stone in regulating the expression of circRNA in response to the externally added small molecules, presenting one possible strategy to regulate circRNA biogenesis. Recent progress in molecular designs that target particular RNA motifs should accelerate the discovery of RNA-binding small molecules that can regulate the biogenesis of biologically important circRNAs.

\section{Conflicts of interest}

There are no conflicts to declare.

\section{Notes and references}

1 W. R. Jeck and N. E. Sharpless, Nat. Biotechnol., 2014, 32, 453-461. 2 L. S. Kristensen, M. S. Andersen, L. V. W. Stagsted, K. K. Ebbesen, T. B. Hansen and J. Kjems, Nat. Rev. Genet., 2019, 20, 675-691.
3 C. Cocquerelle, B. Mascrez, D. Hétuin and B. Bailleul, FASEBJ., 1993, $7,155-160$.

4 Z. Pasman, M. D. Been and M. A. Garcia-Blanco, RNA, 1996, 2, 603-610.

5 S. Memczak, M. Jens, A. Elefsinioti, F. Torti, J. Krueger, A. Rybak, L. Maier, S. D. Mackowiak, L. H. Gregersen, M. Munschauer, A. Loewer, U. Ziebold, M. Landthaler, C. Kocks, F. Le Noble and N. Rajewsky, Nature, 2013, 495, 333-338.

6 A. Rybak-Wolf, C. Stottmeister, P. Glažar, M. Jens, N. Pino, S. Giusti, M. Behm, O. Bartok, R. Ashwal-Fluss, M. Herzog, L. Schreyer, P. Papavasileiou, A. Ivanov, M. Öhman, D. Refojo, S. Kadener and N. Rajewsky, Mol. Cell, 2014, 58, 870-885.

7 E. W. Ottesen, D. Luo, J. Seo, N. N. Singh and R. N. Singh, Nucleic Acids Res., 2019, 47, 2884-2905.

8 J. Salzman, R. E. Chen, M. N. Olsen, P. L. Wang and P. O. Brown, PLoS Genet., 2013, 9, e1003777.

9 P. G. Maass, P. Glažar, S. Memczak, G. Dittmar, I. Hollfinger, L. Schreyer, A. V. Sauer, O. Toka, A. Aiuti, F. C. Luft and N. Rajewsky, J. Mol. Med., 2017, 95, 1179-1189.

10 M. Piwecka, P. Glažar, L. R. Hernandez-Miranda, S. Memczak, S. A. Wolf, A. Rybak-Wolf, A. Filipchyk, F. Klironomos, C. A. Cerda Jara, P. Fenske, T. Trimbuch, V. Zywitza, M. Plass, L. Schreyer, S. Ayoub, C. Kocks, R. Kühn, C. Rosenmund, C. Birchmeier and N. Rajewsky, Science, 2017, 357, 6357.

11 Q. Zheng, C. Bao, W. Guo, S. Li, J. Chen, B. Chen, Y. Luo, D. Lyu, Y. Li, G. Shi, L. Liang, J. Gu, X. He and S. Huang, Nat. Commun., 2016, 7, 11215.

12 T. B. Hansen, T. I. Jensen, B. H. Clausen, J. B. Bramsen, B. Finsen, C. K. Damgaard and J. Kjems, Nature, 2013, 495, 384-388.

13 R. Ashwal-Fluss, M. Meyer, N. R. Pamudurti, A. Ivanov, O. Bartok, M. Hanan, N. Evantal, S. Memczak, N. Rajewsky and S. Kadener, Mol. Cell, 2014, 56, 55-66.

14 C. X. Liu, X. Li, F. Nan, S. Jiang, X. Gao, S. K. Guo, W. Xue, Y. Cui, K. Dong, H. Ding, B. Qu, Z. Zhou, N. Shen, L. Yang and L. L. Chen, Cell, 2019, 177, 865.

15 N. R. Pamudurti, O. Bartok, M. Jens, R. Ashwal-Fluss, C. Stottmeister, L. Ruhe, M. Hanan, E. Wyler, D. Perez-Hernandez, E. Ramberger, S. Shenzis, M. Samson, G. Dittmar, M. Landthaler, M. Chekulaeva, N. Rajewsky and S. Kadener, Mol. Cell, 2017, 66, 9-21.

16 J. N. Vo, M. Cieslik, Y. Zhang, S. Shukla, L. Xiao, Y. Zhang, Y.-M. Wu, S. M. Dhanasekaran, C. G. Engelke, X. Cao, D. R. Robinson, A. I. Nesvizhskii and A. M. Chinnaiyan, Cell, 2019, 176, 869.

17 J. Bi, H. Liu, W. Dong, W. Xie, Q. He, Z. Cai, J. Huang and T. Lin, Mol. Cancer, 2019, 18, 1-14.

18 U. Dube, J. L. Del-Aguila, Z. Li, J. P. Budde, S. Jiang, S. Hsu, L. Ibanez, M. V. Fernandez, F. Farias, J. Norton, J. Gentsch, F. Wang and S. Salloway, et al., Nat. Neurosci., 2019, 22, 1903-1912.

19 L. Kumar, Shamsuzzama, R. Haque, T. Baghel and A. Nazir, Mol. Neurobiol., 2017, 54, 7224-7234.

20 Y. Lee and D. C. Rio, Annu. Rev. Biochem., 2015, 84, 291-323.

21 X. Li, S. Liu, L. Zhang, A. Issaian, R. C. Hill, S. Espinosa, S. Shi, Y. Cui, K. Kappel, R. Das, K. C. Hansen, Z. H. Zhou and R. Zhao, Nature, 2019, 573, 375-380.

22 D. Liang and J. E. Wilusz, Genes Dev., 2014, 28, 2233-2247.

23 X. O. Zhang, H. Bin Wang, Y. Zhang, X. Lu, L. L. Chen and L. Yang, Cell, 2014, 159, 134-147.

24 L.-L. Chen, Nat. Rev. Mol. Cell Biol., 2016, 17, 205-211.

25 W. R. Jeck, J. A. Sorrentino, K. Wang, M. K. Slevin, C. E. Burd, J. Liu, W. F. Marzluff and N. E. Sharpless, $R N A$, 2013, 19, 141-157.

26 M. Wang, J. Hou, M. Müller-McNicoll, W. Chen and E. M. Schuman, iScience, 2019, 20, 237-247.

27 R. Yoshimoto, K. Rahimi, T. B. Hansen, J. Kjems and A. Mayeda, iscience, 2020, 23, 101345.

28 T. Peng and K. Nakatani, Angew. Chem., Int. Ed., 2005, 44, 7280-7283.

29 T. Peng, C. Dohno and K. Nakatani, Angew. Chem., Int. Ed., 2006, 45, 5623-5626.

30 T. Shibata, K. Nagano, M. Ueyama, K. Ninomiya, T. Hirose, Y. Nagai, K. Ishikawa, G. Kawai and K. Nakatani, Nat. Commun., 2021, 12, 236.

31 A. C. Panda, K. Abdelmohsen and M. Gorospe, Methods Mol. Biol., 2017, 1534, 79-87.

32 S. Lefever, F. Pattyn, J. Hellemans and J. Vandesompele, Clin. Chem., 2013, 59, 1470-1480.

33 K. J. Livak and T. D. Schmittgen, Methods, 2001, 25, 402-408. 